\title{
Grazer-induced transcriptomic and metabolomic response of the chain-forming diatom Skeletonema marinoi
}

\author{
Alberto Amato $\mathbb{1}^{1,7}$. Valeria Sabatino ${ }^{1}$ - Göran M. Nylund ${ }^{2}$. Johanna Bergkvist ${ }^{3}$ - Swaraj Basu ${ }^{1,8}$. \\ Mats X. Andersson ${ }^{3} \cdot$ Remo Sanges $\mathbb{C}^{4} \cdot$ Anna Godhe ${ }^{5} \cdot$ Thomas Kiørboe $^{6} \cdot$ Erik Selander $^{5} \cdot$ Maria I. Ferrante $\mathbb{C}^{1}$
}

Received: 4 August 2017 / Revised: 22 November 2017 / Accepted: 25 January 2018 / Published online: 29 March 2018

(c) The Author(s) 2018. This article is published with open access

\begin{abstract}
Diatoms and copepods are main actors in marine food webs. The prey-predator interactions between them affect bloom dynamics, shape marine ecosystems and impact the energy transfer to higher trophic levels. Recently it has been demonstrated that the presence of grazers may affect the diatom prey beyond the direct effect of grazing. Here, we investigated the response of the chain-forming centric diatom Skeletonema marinoi to grazer cues, including changes in morphology, gene expression and metabolic profile. S. marinoi cells were incubated with Calanus finmarchicus or with Centropages typicus and in both cases responded by reducing the chain length, whereas changes in gene expression indicated an activation of stress response, changes in the lipid and nitrogen metabolism, in cell cycle regulation and in frustule formation. Transcripts linked to $G$ protein-coupled receptors and to nitric oxide synthesis were differentially expressed suggesting involvement of these signalling transduction pathways in the response. Downregulation of a lipoxygenase in the transcriptomic data and of its products in the metabolomic data also indicate an involvement of oxylipins. Our data contribute to a better understanding of the gene function in diatoms, providing information on the nature of genes implicated in the interaction with grazers, a crucial process in marine ecosystems.
\end{abstract}

\section{Introduction}

Predation is the largest source of mortality for marine phytoplankton with $>90 \%$ of the pelagic primary production consumed by zooplankton $[1,2]$. The pelagic lifestyle offers a limited repertoire of anti-predator strategies compared

Electronic supplementary material The online version of this article (https://doi.org/10.1038/s41396-018-0094-0) contains supplementary material, which is available to authorised users.

Maria I. Ferrante

mariella.ferrante@szn.it

1 Integrative Marine Ecology, Stazione Zoologica Anton Dohrn, Villa Comunale 1, Naples 80121, Italy

2 Department Marine Sciences, University of Gothenburg, Tjärnö, SE-452 96 Strömstad, Sweden

3 Department of Biology and Environmental Sciences, University of Gothenburg, Box 461, SE-405 30 Göteborg, Sweden

4 Biology and Evolution of Marine Organisms Stazione Zoologica Anton Dohrn, Villa Comunale 1, Naples 80121, Italy

5 Department Marine Sciences, University of Gothenburg, with more complex benthic and terrestrial habitats. Yet, pelagic organisms can modulate key traits to reduce predation risk. For example, some species of phytoplankton may modify chain length, swimming speed or toxin production in response to the presence of predators, which reduce predator encounter rates or palatability and, hence, predation mortality [3-8]. Behavioural responses to the presence of predators may cascade through food webs and may have larger impact on food web structure than predation rates themselves [9]. Adaptive responses to the threat from consumers are present in organisms from bacteria to

Box 461, SE-405 30 Gothenburg, Sweden

6 Centre for Ocean Life, DTU-Aqua Kemitorvet Building 202, 2800 Kgs, Lyngby, Denmark

7 Present address: Laboratoire de Physiologie Cellulaire et Végétale, UMR5168 CNRS-CEA-INRA-Université de Grenoble Alpes, Institut de Recherche en Science et Technologies pour le Vivant, CEA Grenoble, 17 rue des Martyrs, 38054 Grenoble Cédex 9, France

8 Present address: Department of Medical Biochemistry and Cell Biology, Institute of Biomedicine, Sahlgrenska Academy, University of Gothenburg, SE-405 30 Gothenburg, Sweden 
large vertebrates [10] and are known to cause cascading effects in food webs [11].

Diffusible cues from zooplankton predators trigger changes in key traits serving to evade consumers with potential impact on food web structure [12]. These processes have received little attention in phytoplankton ecology, and the molecular mechanism through which microphytoplankton sense and respond to consumers has been examined in very few studies. Alexandrium minutum, a harmful dinoflagellate, increases the production of paralytic shellfish toxins in the presence of copepods through the regulation of relatively few genes [13]. Most of the regulated genes have unknown function, making it difficult to reconstruct a mechanism for the response. Transcriptomics allowed the exploration of the differences in gene expression between two Alexandrium fundyense strains exposed to a protist grazer [14]. Signal transduction via $\mathrm{G}$ protein-coupled receptors (GPCRs) was hypothesised in A. fundyense when exposed to the heterotrophic dinoflagellate, but DNA methylation and thus epigenetic regulation was also indicated.

Here, we consider the predator-prey interaction between copepods and diatoms. Copepods are among the most successful and abundant multicellular organisms in the sea and among the main grazers of diatoms and other microphytoplankton in the ocean. Diatoms are unicellular photosynthetic protists that have colonised almost all humid environments, and represent one of the major groups of marine primary producers.

Several diatom species produce polyunsaturated aldehydes and other fatty acid (FA) derivatives that lead to reduced fecundity and induce larval malformations in copepods that fed on them [15]. Among these, the ubiquitous marine colonial, bloom-forming diatom Skeletonema marinoi [16], known to produce several oxygenated FA derivatives, including different classes of oxylipins [17]. These compounds have been proposed to play a role in allelopathy and cell-to-cell signalling [18-20].

We have previously shown that $S$. marinoi modifies its colony size in response to chemical cues from copepods [7]. Here, we aim at identifying the molecular mechanism of diatom response to the presence of copepod grazers by comparing metabolic and gene expression responses. We apply a multiomics approach, targeting small molecules, FA composition and transcriptional changes simultaneously. By using this approach we identify the genes involved in threat detection and the onset of a smaller colony size phenotype. We observe the induction of a stress response, changes in the lipid and nitrogen metabolisms, and a response in genes possibly associated with division and chain dynamics. We also highlight changes in signal transduction pathways, such as signalling mediated by GPCRs, one of the largest classes of cell-surface receptors involved in a wide range of

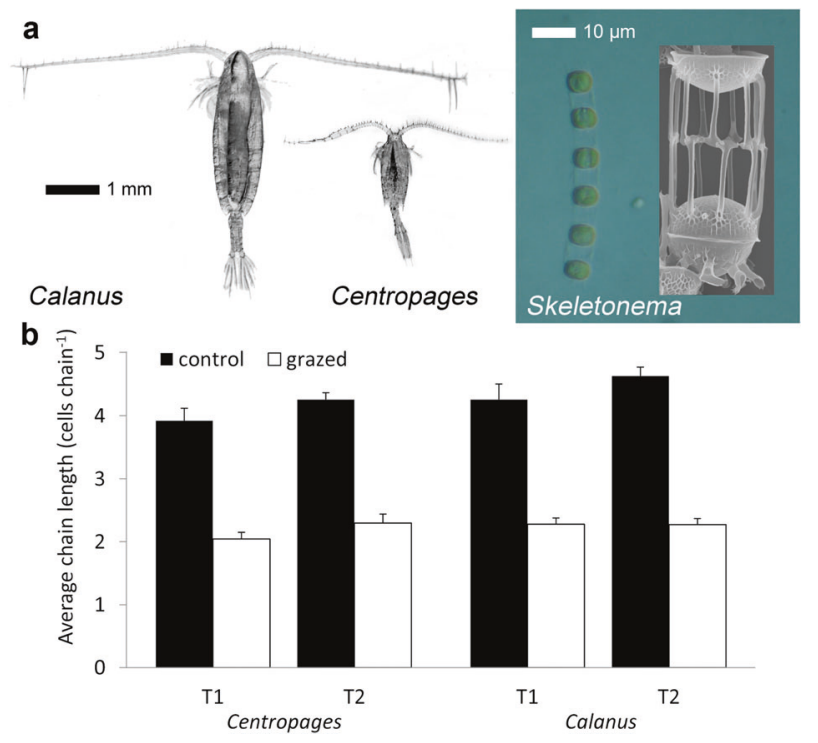

Fig. 1 a The calanoid grazers Calanus finmarchicus and Centropages typicus were used in bottle incubations with Skeletonema marinoi, the chain-forming diatom depicted in the top right light micrograph. The inset electron micrograph details the connection between daughter cells, which is split up in response to copepod grazers. The white scale bar applies to the electron micrograph. b Chain length of Skeletonema marinoi after 65 and $89 \mathrm{~h}$ (T1 and T2) of exposure to Calanus finmarchicus or Centropages typicus. Grazer-free controls are shown in black, and grazer-exposed cultures in white. Both grazers induced a similar and significant shortening of chains. Bars show average values of three replicates, error bars denote standard error of mean

processes [21]. The metabolomics data extend the gene expression data and indicate complex changes occurring downstream of the transcriptomic level of gene regulation.

\section{Materials and methods}

\section{Organisms and experimental design}

Females of the copepods Centropages typicus and Calanus finmarchicus (Fig. 1a) were picked from plankton samples collected near Tjärnö Island in the North Sea (Sweden; 58 $\left.\mathrm{N} 51^{\prime}-11^{\circ} \mathrm{E} 7^{\prime}\right)$ and maintained in filtered sea water until the next morning when experiments started. S. marinoi strain $\mathrm{V} 32$, isolated from a germinated resting stage originating from surface sediment near the Vinga island in the Skagerrak, was cultivated in F2 medium [22] in 2 1-Pyrex ${ }^{\circledR}$ sterile bottles and kept in exponential growth by daily dilutions until use. Photoperiod and irradiance were 16L:8D and $\sim 80 \mu \mathrm{mol}$ photos $\mathrm{m}^{-2} \mathrm{~s}^{-1}$.

Two experiments were run, one with Centropages typicus (Exp.1) and one with Calanus finmarchicus (Exp.2). At time 0, copepods (7 C. typicus or 10 C. finmarchicus) were added to each of 10 2-litre bottles, leaving another 10 bottles as ungrazed controls. Five grazed and un-grazed bottles were harvested after $65 \mathrm{~h}$ and the remaining five after $89 \mathrm{~h}$ from 
introducing animals into the bottles ( $\mathrm{T} 1$ and $\mathrm{T} 2$, respectively) (Supplementary Figure 1). Sampling points were chosen based on the effects seen in Bergkvist et al. [7]. At each time point, $15 \mathrm{ml}$ were fixed for microscopic cell counts; $50 \mathrm{ml}$ were analysed by microscopy to obtain chain length spectra; $5 \mathrm{ml}$ were used for the Coulter Counter (Beckman); 1 litre was filtered to isolate RNA and $500 \mathrm{ml}$ to isolate metabolites. Copepods were excluded from the last two samples by prefiltering through a sieve $(64 \mu \mathrm{m}$ mesh size, this allows even the longest diatom chains to pass through). For RNAseq, three controls and three treated samples for each time point in Exp.2 were used. RNA extraction and sequencing are described in Supplementary File 1.

\section{qPCR validations}

For quantitative PCR (qPCR) validations, 12 RNA samples (three controls and three copepod-treated for each time point) from Exp. 1 and 8 samples (two controls and two copepod-treated for each time point) from Exp.2 were reverse transcribed using Qiagen QuantiTect Reverse Transcription Kit (Qiagen). qPCR was conducted as described in Basu et al. [23] using an Applied Biosystems ViiA $^{\text {TM }} 7$ Real-Time PCR System (Life Technologies).

\section{Reference transcriptome generation and annotation}

The raw reads (accession E-MTAB-6076) were adapter filtered and quality trimmed using Trimmomatic [24] then assembled using Trinity [25] and annotated using Annocript pipeline [26] (Supplementary File 1). The expression levels were quantified using Bowtie [27]. Transcripts $<200$ nucleotides or with an expression $<1$ count per million (CPM) were removed.

\section{Phylogenetic analyses}

All the sequence data sets (Supplementary File 1) were automatically aligned using ClustalW implemented in MEGA6 software [28] and the alignment was then manually curated. Bestfit [29] defined the best evolution model (the best model is reported in the figure legends). For maximum likelihood (ML) analyses, 10,000 bootstrap replicates were performed.

\section{Liquid cromatography-mass spectrometry (LC-MS) analysis, processing and multivariate analysis}

Samples were analysed on an Agilent 1100 HPLC coupled to an Agilent Q-TOF 6540 mass spectrometer. The samples were separated on a Licrosphere $2.1 \times 150 \mathrm{~mm}, 3 \mu \mathrm{m}$ C18 silica column (Poroshell, Agilent) using an eluent gradient. The resulted raw data were processed and aligned to generate a list of features (hereafter referred to as "metabolites") consisting of retention time and molecular masses. A detailed description of the analyses and processing is given in Supplementary File 1. Calculations of elemental compositions were only performed on metabolites with a neutral mass lower than $500 \mathrm{Da}$. Data were normalised by biovolume from electronic cell counts before multivariate analysis to avoid confounding effects of grazing. The metabolic response of Skeletonema to copepod grazing was analysed with multivariate data analysis, using the software SIMCA (version 13.0.0.0, Umetrics, Sweden). Principal component analysis was used to generate an overview and to analyse for systematic differences in metabolite content between treatments. The metabolite data were modelled and interpreted using orthogonal partial least squares with discriminant analysis [30, 31]. Data have been deposited to MetaboLights (MTBLS571) [32].

\section{Total FA analysis}

Total FAs were extracted and methanolised as described in Supplementary File 1, then resuspended in heptane and analysed using an Agilent 7820 GC coupled to an Agilent 5975 mass selective detector. The FA methyl esters were separated on a $30 \mathrm{~m}, 0.25 \mathrm{~mm}$ DB-23 capillary column (Agilent) using helium as carrier gas at a constant flow of $0.6 \mathrm{ml} \mathrm{min}^{-1}$. Methyl esters were identified by mass spectrum and compared with authentic standards (Me 100, Me81 and individual FA methyl esters, Larodan, Solna, Sweden and Marine PUFA no.3, Sigma-Aldrich). The FA methyl esters were quantified from the total ion chromatogram against the 19:0 internal standard.

\section{Results}

\section{Diatom chain length reduction in presence of copepods}

S. marinoi cells grown in the presence or absence of the copepods Centropages typicus or Calanus finmarchicus differed in cell chain length: cell concentrations were lower and chain lengths were shorter in the copepod-exposed cultures compared with the controls (Table 1, Fig. 1, Supplementary Figure 2).

\section{General gene expression profile and metabolite changes in diatoms exposed to copepods}

\section{RNA-seq profiling of transcriptomic changes induced by grazing}

A total of 4185 genes were differentially expressed in copepod-treated cells compared with control cells (Exp.2). 
Table 1 S. marinoi cell concentration $\left(\right.$ cell $\mathrm{ml}^{-1}$ ) in control and copepod-containing cultures

\begin{tabular}{llll}
\hline & Start & T1—after 65h & T2-after 89h \\
\hline Exp.1 control & 5000 & $27,510 \pm 2748^{*}$ & $44,920 \pm 4953$ \\
Exp.1 Centropages typicus treated & 5000 & $15,563 \pm 4006^{*}$ & $24,050 \pm 3143$ \\
Exp.2 control & 10,000 & $46,822 \pm 3799$ & $114,373 \pm 31,244$ \\
Exp.2 Calanus finmarchicus treated & 10,000 & $36,167 \pm 5999$ & $62,104 \pm 17,829$ \\
\hline
\end{tabular}

Average \pm standard deviation is reported $(n=5)$

$* n=4$

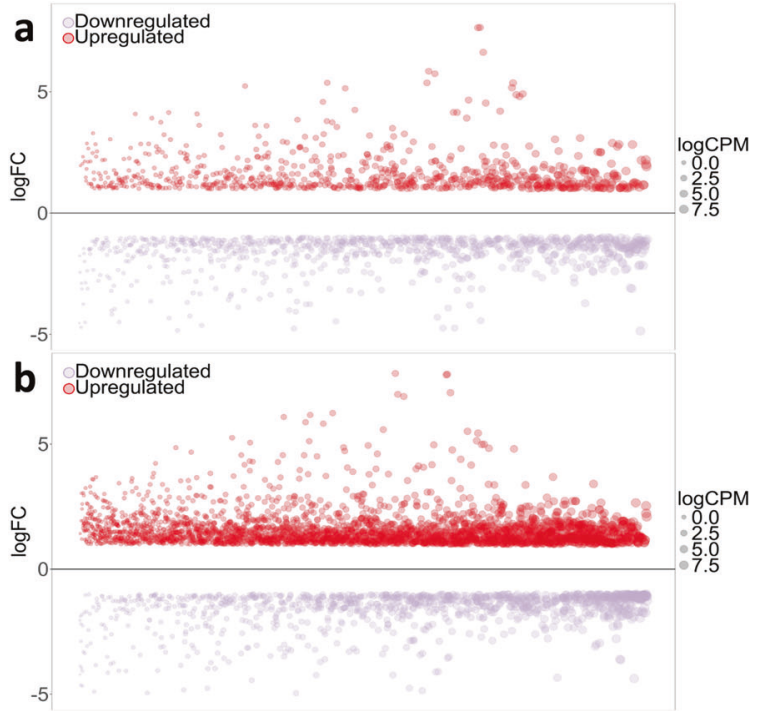

Fig. 2 a, b Plots showing the genes differentially expressed at T1 and $\mathrm{T} 2$, respectively. Fold changes in log scale are reported on the $y$ axis, each dot represents a transcript, the dot size is proportional to the levels of expression measured as CPM (counts per million). Transcripts are arranged on the $x$ axis according to their $\log$ CPM. $\mathbf{c}$ Venn diagram showing the number of shared and unique differentially expressed transcripts at the two time points. d Representation of GO terms significantly enriched among differentially expressed transcripts.

The expression of $7 \%$ and $16.5 \%$ of the total S. marinoi transcripts were regulated at $\mathrm{T} 1$ and $\mathrm{T} 2$, respectively, indicating a stronger response at the second time point (Supplementary Tables S5-S6, Fig. 2a-b). Almost 2/3 of the differentially expressed genes could be associated with a known function (a SwissProt, Conserved Domain or UniRef description).

A total of 509 genes were downregulated and 598 genes were upregulated at both time points, whereas only 6 genes were downregulated at $\mathrm{T} 1$ and upregulated at $\mathrm{T} 2$ (Fig. 2c): two unknown, a putative oxidoreductase, a High-affinity nitrate transporter 2.2, Fibrocystin- $L$ and Glutamine synthetase.

We looked for functions that appeared in the differentially expressed genes with a higher frequency than in the entire transcriptome (gene ontology (GO) and pathway enrichments, Fig. 2d, Supplementary Tables S7-S8). Cells appeared to be mainly rewiring transcriptional regulation at $\mathrm{T} 1$, whereas at $\mathrm{T} 2$ there seemed to be an established

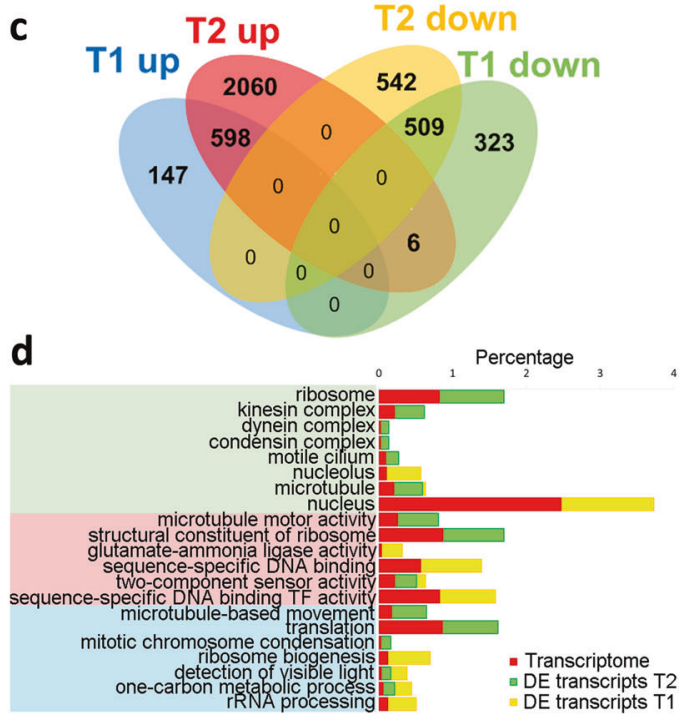

The charts show the proportion of transcripts associated with the significant classes among differentially expressed transcripts at $\mathrm{T} 1$ (yellow) and T2 (green) and their respective proportion in the assembled reference transcriptome (red). On $x$ axis, percentage is reported. On $y$ axis, GO terms. The green shade indicates GO terms associated with cellular component, the purple to molecular function and the blue to biological processes

transcriptional programme mainly involving translation and energy metabolism.

Notably, we observed 9 and 36 long non-coding RNAs to be differentially regulated in copepod-exposed samples at $\mathrm{T} 1$ and $\mathrm{T} 2$, respectively, indicating their participation in regulating cellular response in the presence of grazers.

\section{Validation of the transcriptional response using $\mathrm{QPCR}$}

To validate the RNA-seq results, we selected 10 differentially expressed genes representing different pathways and performed qPCR analyses on independent grazed and control cultures from Exp.2 (Table 2). The Pearson's correlation between RNA-seq and qPCR results for Exp.2 indicated high consistency $\left(R^{2}=0.95, p=2.3 \mathrm{e}-05\right.$ for T1 and $R^{2}=0.89, p=0.0006$ for T2). The same set of genes was tested on RNA samples produced from Exp. 1 and the results were similar (Table 2$)\left(R^{2}=0.80, p=0.0048\right.$ at T1 
Table 2 Validation of the transcriptional response with qPCR

\begin{tabular}{|c|c|c|c|c|c|c|c|}
\hline \multirow[t]{3}{*}{ Transcript ID } & \multirow[t]{3}{*}{ Gene function } & \multicolumn{6}{|c|}{$\operatorname{LogFC}$} \\
\hline & & \multicolumn{2}{|c|}{ RNA-seq Exp2 } & \multicolumn{2}{|c|}{$\begin{array}{l}\text { qPCR Exp1 } \\
\text { Centropages }\end{array}$} & \multicolumn{2}{|c|}{$\begin{array}{l}\text { qPCR Exp2 } \\
\text { Calanus }\end{array}$} \\
\hline & & $\mathrm{T} 1$ & $\mathrm{~T} 2$ & $\mathrm{~T} 1$ & $\mathrm{~T} 2$ & $\mathrm{~T} 1$ & $\mathrm{~T} 2$ \\
\hline c13275_g2_i2 & Heat stress transcription factor $\mathrm{A}-4 \mathrm{~b}$ & 1.8 & 1.7 & 3.1 & 3.3 & 5 & 4.6 \\
\hline c7746_g1_i1 & cAMP-dependent protein kinase type 1 & -3.8 & -4.1 & -1.7 & -2.7 & -3.7 & -3.2 \\
\hline c6906_g1_i1 & Peroxiredoxin Q & -2.5 & -2.6 & -1 & -2.6 & -2.9 & -2.2 \\
\hline c9427_g2_i1 & no annotation & 5.8 & 8.2 & 3.4 & 2.2 & 5.9 & 5.2 \\
\hline c20406_g1_i1 & no annotation & -2.6 & -2.9 & -1.6 & -1.2 & -2.8 & -2.4 \\
\hline c16128_g1_i1 & Phospholipase A1-Igamma2 & -2 & -1.4 & -2.2 & -1.7 & -2.8 & 0.1 \\
\hline c8316_g1_i1 & Chitinase A1 & -2.7 & -2.5 & 1.6 & -1.5 & -3.7 & -2.5 \\
\hline c5256_g1_i1 & Phosphatidylinositol 3-kinase TOR2 & - & 1.1 & 0.5 & 0.5 & -0.1 & 3.8 \\
\hline c9108_g1_i1 & Phosphoacetylglucosamine mutase & -1.2 & -1.5 & -0.8 & 0.5 & -2.1 & -1.9 \\
\hline c12337_g1_i1 & $\begin{array}{l}\text { Glutamine-fructose-6-phosphate } \\
\text { aminotransferase }\end{array}$ & -2.3 & -2.8 & -1.6 & 0.3 & -3.2 & -2.6 \\
\hline
\end{tabular}

LogFC (fold change) is reported for each transcript on sampling occasions T1 and T2 for RNA-seq from Exp2 and qPCR analyses for Experiment 1 (Centropages typicus) and Experiment 2 (Calanus finmarchicus). The qPCR values, which are in accordance with the RNA-seq values, are in boldface

and $R^{2}=0.74, p=0.0145$ at T2). Thus, $S$. marinoi cells exhibit analogous responses to the presence of two different copepod species.

\section{Metabolomics}

In total, we detected 769 metabolites that were present in at least three replicates within a single treatment group. Out of these, 242 metabolites were present at different concentrations in grazed and control cultures (Fig. 3, Supplementary Figures 3-4, Supplementary Table S9). In contrast to the transcriptomics data, the difference between grazed and control cultures was larger at T1. There were more downregulated than upregulated metabolites in grazed cultures at T1 and the majority of downregulated metabolites at T2 were also downregulated at T1 (Fig. 3a, Supplementary Table S9). Upregulated metabolites were less consistent between time points, with the proportion of shared upregulated metabolites constituting $30 \%$ and $40 \%$ at $\mathrm{T} 1$ and $\mathrm{T} 2$, respectively. The treatment effect at $\mathrm{T} 1$ was mainly due to upregulation of larger molecular weight metabolites in grazed treatments, indicative of anabolism. The targeted analysis of FA showed a relative increase of longer chain polyunsaturated FA in grazed treatments at the expense of shorter chain FA (Supplementary Figure 5). At T2, no clear distinction in molecular size between upregulated and downregulated metabolites was recorded.

\section{Signal transduction}

The perception of external cues must trigger a signal transduction cascade and this prompted a search for transcripts related to this process. $S$. marinoi cells appear to transduce signals from the copepods through the GPCR signalling pathway. Transcript c19949_g1_i1 was 1.6-fold higher in copepod-treated samples and finds its best diatom homologue in the Phaeodactylum tricornutum GPCR3 (XP_002186461 [33]). In addition, different transcripts encoding proteins belonging to GPCRs signal transduction routes were regulated: at T2, a putative adenylate cyclase (c6665_g1_i1) and cyclic nucleotide-gated cation channels were upregulated, as was a putative phosphatidylinositol 3kinase (c16565_g1_i1). GPCR signalling can also proceed via Rho GTPases. As the GTPase family is complex, we constructed a phylogenetic tree for small GTPases in $S$. marinoi (Supplementary Figure 6), and found that some of the family members were regulated in our data set. Upregulation of a tubby-like protein (transcript c11030_g1_i1), and of mitogen-activated protein kinase (MAPK) and mitogen-activated protein kinase kinase (MAPKK), downstream of GPCRs and associated with cell proliferation, were also found.

Nitric oxide (NO) signalling may also be implicated in the response to grazers, as a gene encoding a NO synthase was upregulated (c9307_g1_i1, corresponding to MMETSP1040-20121108I15944 [34]).

\section{Stress response}

Transcripts annotated as heat shock factor proteins or heat stress transcription factors, belonging to the enriched term sequence-specific DNA binding (Fig. 2d), were among those showing the highest changes (Supplementary Tables S5S6). Other signatures of stress were the upregulation of 
a

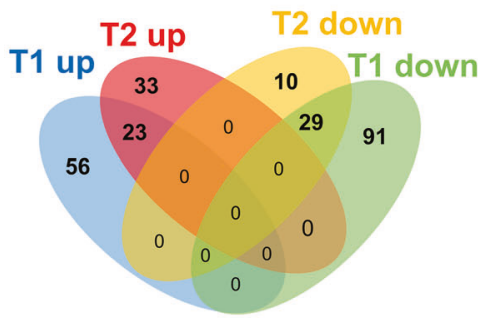

b

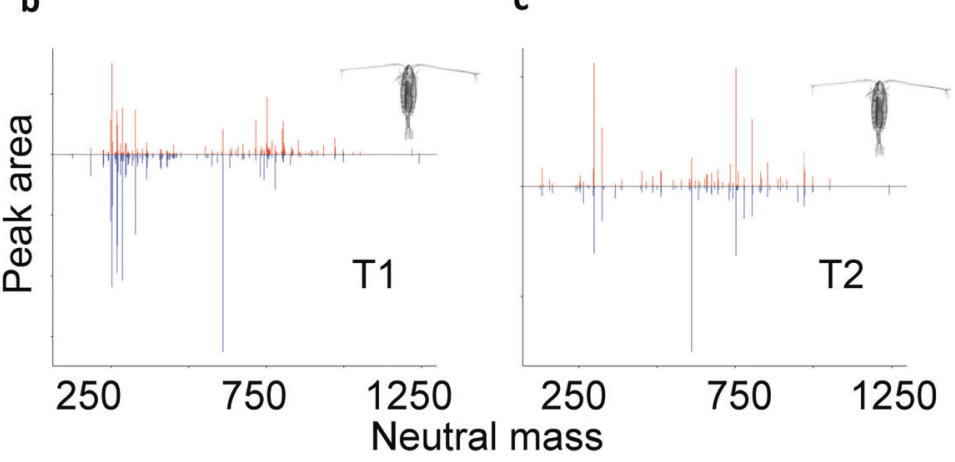

Fig. 3 a The Venn diagram shows the number of metabolites that are commonly regulated (up or down) at both occasions, as well as metabolites upregulated, or downregulated on a single occasion. No metabolites showed opposite regulation between $\mathrm{T} 1$ and $\mathrm{T} 2$. b, c
Mirror plots showing the abundance of the individual compounds with copepod samples projected upwards in red and control samples mirrored downward in blue. The increased proportion of larger metabolites in copepod-treated cells is clear in $\mathbf{b}$, but less so in $\mathbf{c}$
Cytochrome P450, and of a Retrovirus-related Pol polyprotein, suggesting activation of transposable elements in response to grazing. A few fucoxanthin and chlorophyll binding proteins were downregulated at both time points, indicating a possible reduction of the photosynthetic activity in cells exposed to copepods.

\section{Lipid and nitrogen metabolism}

Lipid and nitrogen metabolisms appeared to be impacted by the presence of copepods, with a general tendency to degrade complex lipids and to increase nitrate uptake. Pathway enrichment analyses showed Lipid metabolism and $F A$-oxidation to be significantly enriched in the differentially expressed genes data sets (Supplementary Table S8).

Genes encoding enzymes involved in subsequent steps of FA oxidation were all upregulated at both time points (Supplementary Figure 7). We also found upregulation of an acyl-CoA carboxylase (c13306_g1_i1) that converts acetyl-CoA into malonyl-CoA in the first committed step of FA biosynthesis. Acyl-CoA carboxylases can have different subcellular localisation, using a signal peptide prediction software [35] we found a predicted chloroplastic localisation for the c13306_g1_i1 protein product. At T1, two putative FA desaturases ( -1.8 and -1.9 -fold), a phospholipase A (-2.0) and a lipoxygenase (-2.4) were downregulated, suggesting that lipid secondary metabolism is affected as well (Supplementary Table S5).

Lipoxygenases catalyse the production of lipid derivatives called oxylipins. Several downregulated compounds in the grazed treatment at $\mathrm{T} 1$ had retention times and predicted elemental compositions consistent with oxylipins, in line with the gene expression changes indicating a downregulation of this pathway.

As the pathway analysis implicated the FA metabolism in the response to copepods (Supplementary Table S8), we complemented the metabolic profiling with total FA profiling (Supplementary Figure 5). Eicosapentaenoic acid (EPA, 20:5) consistently had higher relative abundance in grazed cultures, at the expense of shorter chain FA such as 14:0, 16:2, 16:3, 16:4 and 18:4.

At T2, we observed an upregulation of genes involved in nitrogen metabolism [36], generally induced during nitrogen starvation (Supplementary Table S10), possibly due to the presence of nitrogen forms excreted by copepods [37].

\section{Cell cycle and frustule dynamics}

Because grazer cues induce shorter cell chains and as chain formation and splitting is linked to cell division, we searched for transcripts related to cell division. GO enrichments included a number of terms related to cytoskeleton rearrangements and mitosis (Fig. 2d).

Nine cyclin genes, fundamental in the control of the cell cycle, were regulated in response to copepods. One cyclin gene is the homologue of CYCB1 [38]. The other eight regulated $S$. marinoi cyclins were part of the diatom-specific group ( $d s C Y C$, diatom-specific cyclins) [39]. Correspondence of the cyclins regulated in this study to those of the model diatoms $P$. tricornutum and Thalassiosira pseudonana is shown in Supplementary Table S11. Putative $d s C Y C 5, d s C Y C 7$ and $d s C Y C 9$ were downregulated, whereas putative $d s C Y C 6$ and $d s C Y C 3$ were upregulated.

Interestingly, the early steps of chitin biosynthesis were downregulated (Supplementary Figures 8-9). Of three transcripts containing the glutamine:fructose-6-phosphate aminotransferase (GFA1) domain (c10266, GFAla, c12337, GFA1b and c11460g2i1, GFA2, Supplementary Figure 8), GFAla and GFAlb were downregulated at both time points, as were phosphoacetylglucosamine mutase (PAGM), a one-nucleotide sugar transporter and chitinase transcripts (c8316_g1_i1, c6786_g1_i2, c12572_g1_i1). 


\section{Discussion}

The concept that species interactions via chemical signals are a major driving force in shaping the structure of plankton communities has recently found experimental support $[14,40]$. However, data explaining the molecular mechanisms underlying these processes are still scarce.

Bergkvist et al. [7] investigated diatom responses to different copepod species and demonstrated that, in the presence of copepod grazers, S. marinoi reduces chain length in order to escape grazing pressure. Bergkvist et al. [7] carried out both caged and free copepod experiments and observed the reduction in chain length in both cases. The trigger for diatoms to reduce their chain length is the presence of an active grazer. Indeed, caged copepods feeding on a different phytoplankton species, the flagellate Rhodomonas salina produced the same response. Conversely, incubating $S$. marinoi together with the heterotrophic dinoflagellate Gyrodinium dominans or with caged copepods without any prey, did not produce any chain length variation. In the present study, we incubated the same diatom species with two different calanoid copepods. As cages cause a strong signal dampening effect [41], we conducted experiments with free copepods to maximise the stimulus. Although here we cannot clearly separate the effect of grazing, we observed a change in chain length as in previous studies, and analysed the gene expression profile and metabolite composition of cells in the presence of grazers and their cues. The multiomics approach allowed us to start exploring the mechanism mediating the formation of a defence morphotype in Skeletonema.

Temporal changes in gene expression and metabolic profiles suggest substantial metabolic rearrangements. Differences in the transcriptomics and metabolic profiles from grazed and control cultures may partly be affected by a misalignment of the cell cycle transitions between control and grazed cultures. Batch cultures in light-dark cycles tend to divide more or less synchronously. If the grazers triggered a cost that caused delayed or accelerated division in the grazed cultures, it is possible that their cell cycle was displaced relative to controls, which may have contributed to the large metabolic shift seen after $65 \mathrm{~h}$. Different chemical profiles during the different growth phases of $S$. marinoi have been reported, indicating complex changes $[42,43]$. The stronger transcriptomic response observed at $\mathrm{T} 2$ could reflect not only the response to copepod cues but also a reaction to a less crowded culture. Studies in Thalassiosira pseudonana identified a set of functions associated with the exponential state [44], this partially overlaps with the set of functions associated with the grazercontaining (less dense) cultures in our study (Fig. 4). Nevertheless, other functions change in opposite direction (light harvesting photosynthesis for instance), and many regulated transcripts are distinctive of the grazer treatment, as discussed below.

\section{G protein-coupled receptors}

The copepod-produced chemical cues, released in the medium throughout the experiment, must be perceived by the diatoms in order for them to respond. Our results suggest that one possible mechanism responsible for this perception involves GPCRs. GPCRs are components of a large family composed of hundreds of protein subdivided into different classes [45]. All GPCRs sense an extracellular signal and transduce it into a cascade of molecular events [46]. One of the diatom GPCRs and other putative components of the pathway were upregulated in our study (Fig. 4). GPCR signalling can proceed through adenylate cyclase, upregulated in our analyses, with consequent increase in cyclic AMP (cAMP), a second messenger, which activates downstream effectors, such as the cAMPdependent protein kinase and gated channels. GPCR signalling can also proceed via Rho GTPases activation, which can lead to dramatic rearrangements of the cytoskeleton. Signalling through Rho GTPase, which we also observed, might be responsible for the upregulation of transcripts coding for cytoskeletal proteins. Another indication of GPCRs involvement in diatom response to copepods is the upregulation of Tubby-like proteins (TULPs), proteins bound to the membrane phosphoinositol di-phosphate $\left(\mathrm{PIP}_{2}\right)$ that can promote vesicular trafficking upon changes in PIP $_{2}$ mediated by GPCR activation; this triggers TULP dislodgment from the membrane and translocation to the nucleus [47]. In diatoms, vesicular trafficking is involved in frustule deposition through the silica deposition vesicles [48] and this mechanism may thus be associated with chain remodelling in response to copepod predators. Upregulation of MAPK and MAPKK, downstream of GPCRs, is often associated with cell proliferation.

\section{Stress response and NO}

Changes in genes involved in retrotransposition, such as the Retrovirus-related Pol polyprotein found in this study, often mediate retrotransposon mobilisation, a phenomenon associated with the perception of stress cues in diatoms [49].

Major transcriptional changes common to both time points involved a reaction to stressful stimuli, with upregulation of heat shock protein and other stress-responsive genes, including a NO synthase (NOS) gene. NO has been identified as a mediator in a stress surveillance system in diatoms [50]: in P. tricornutum and Thalassiosira weissflogii NO generation was caused by exposure to toxic aldehydes, which in turn were released by damaged cells. $S$. marinoi cells are known to release toxic aldehydes as a 
a

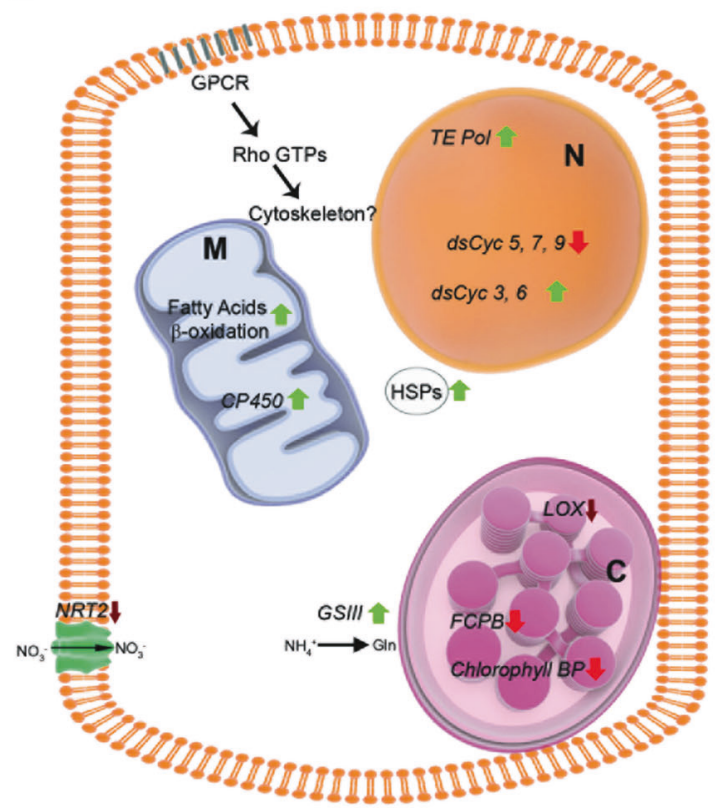

b

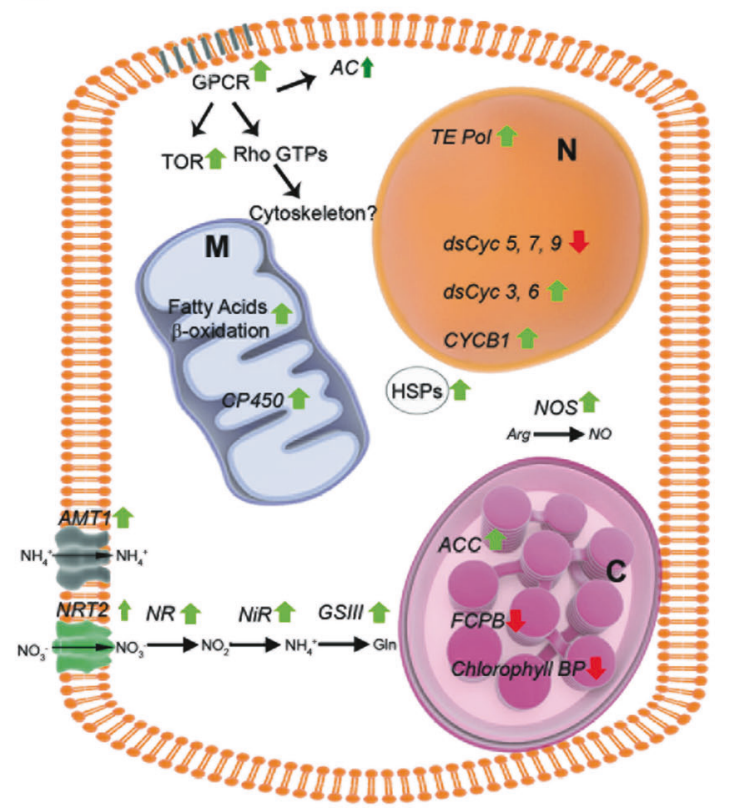

3715 regulated transcripts

95 regulated metabolites

NTR2 nitrate transporter, AMT ammonium transporter, NR nitrate reductase, NiR nitrite reductase, GSIII type-3 glutamine synthetase, HSPs heat shock proteins, NOS nitric oxide synthase, ACC acetylCoA carboxylase, FCPB fucoxanthin-chlorophyll a-c binding protein B

the cytoplasmic ACC. Because of organelle compartmentalisation, malonyl-CoA should remain confined in the chloroplast and should not be mediating any inhibition of the mitochondrial $\beta$-oxidation [52].

FA methyl ester profile indicated that the relative abundance of $\mathrm{C} 14, \mathrm{C} 16$ and $\mathrm{C} 18$ was lower in the grazed treatment, whereas there was an increase of EPA (20:5). In diatoms, triacylglycerols (TAGs) are rich in short chain FA and are almost exclusively stored in lipid droplets [53], whereas EPA is characteristic of the chloroplast lipids like monogalactosyldiacylglycerol and digalactosyldiacylglycerol [54]. The targets of $\beta$-oxidation would be mainly TAGs, so the diminution of short chain FA would mean that lipid droplets are being degraded by the cells for, for example, energy production potentially representing a cost of defence. Desaturases are slow enzymes, hence polyunsaturated FAs accumulate after a certain time in control cultures. If in grazed cultures, cells divide more actively to sustain predation, then a lower amount of 16:4 and 18:4 would be coherent.

\section{Oxylipins}

Oxylipins are FA derivatives with a signalling role in plants and animals, involved in plant defence reactions such as gulation is observed for the chloroplastic ACC and not for 
pathogen infection or wounding [55]. The oxylipin pathway was downregulated at $\mathrm{T} 1$ in the transcriptomics, as well as in the metabolomics data, with a LOX transcript and probable oxylipins downregulated. Cell damage induced by crunching should induce oxylipin production rather than reduction [51]. However, this would occur only in the fraction of cells that is predated and this signal might be diluted. Downregulation of oxylipin production in the entire population might have a different meaning.

\section{Cell division and chain splitting}

Distinctive cyclin genes displayed a change in their expression in grazed cultures. CYCB1, dsCYC6 and $d s C Y C 3$, increased in these cells, are predominantly expressed during the $\mathrm{S}\left(d_{s} C Y C 6\right)$ and the G2/M (CYCB1 and $d s C Y C 3$ ) phases of the cell cycle in P. tricornutum [38, 39]. The downregulated genes $d s C Y C 5, d s C Y C 7$ and dsCYC9 on the other hand have been shown to respond to increases in phosphate and silica concentrations and are supposed to integrate external signals in the cell cycle [38]. These data suggest that cells were responding to a signal and were engaged in active division.

Division and chain formation/splitting involve the assembly and rearrangement of frustules, a process that is still little described in terms of molecular changes. Chain splitting is linked to cell division, which seems to be enhanced in the presence of copepods (cycling and MAPK genes changing and a metabolic profile indicative of anabolic processes). Coherently, signals for cytoskeleton rearrangements were present and, interestingly, changes in genes linked to chitin were also observed. In diatoms, chitin has been hypothesised to be involved in cell wall processes [56] and was found to be associated with the silica cell wall in T. pseudonana [57]. In S. marinoi chains, cells are joined by siliceous connections of the external projections of fultoportulae visible in the scanning electron micrograph in Fig. 1a [58]. Fultoportulae are usually associated with chitin secretion [59] and this biopolymer could be involved in chain formation, possibly by producing an organic template for silica deposition. The downregulation of the chitin synthase pathway can lead to weaker links between adjacent cells when grazers are perceived, with resulting shorter chains formed. Chitin-silica interaction is demonstrated both in vitro and in vivo $[60,61]$ and a possible involvement of chitin in frustule deposition is highly likely.

\section{Conclusions}

Phytoplankton-grazer interactions are key factors in shaping pelagic food web structure, but indirect effects and defence responses mediated by grazer signals, such as the response dissected in our study, may at times be more important than grazing mortality itself in defining ecosystem function [62].

The importance of signalling molecules and of secondary metabolites with infochemical roles in cell-to-cell and trophic interactions has been put forward many times. Here we provide evidence that GPCRs, oxylipins and NO might be an important part of the response of diatoms to copepod cues. Based on previous assumptions, downregulation of oxylipins in the grazed cell population was unexpected, suggesting a complex role for these compounds, which needs to be further clarified. Other changes in the lipid metabolism and in the cell cycle dynamics might indicate that cells modified their division rate in the presence of copepods, most likely increasing divisions, and this might also have impacts on cell distribution and abundance at sea.

Similar results were obtained performing the experiments with two different species of copepods, suggesting a broad significance of our findings.

Acknowledgements We acknowledge the support of the European Community-Research Infrastructure Action under the FP7 "Capacities" Specific Programme, ASSEMBLE grant 227799, the Swedish Research Council VR 2015-05491 (to ES), the European Union under FP7-People, GA n. 600407 (to AA), the Swedish Research Council Formas and VR 217-2008-1719 (to GMN).

\section{Compliance with ethical standards}

Conflict of interest The authors declare that they have no conflict of interest.

Open Access This article is licensed under a Creative Commons Attribution-NonCommercial-ShareAlike 4.0 International License, which permits any non-commercial use, sharing, adaptation, distribution and reproduction in any medium or format, as long as you give appropriate credit to the original author(s) and the source, provide a link to the Creative Commons license, and indicate if changes were made. If you remix, transform, or build upon this article or a part thereof, you must distribute your contributions under the same license as the original. The images or other third party material in this article are included in the article's Creative Commons license, unless indicated otherwise in a credit line to the material. If material is not included in the article's Creative Commons license and your intended use is not permitted by statutory regulation or exceeds the permitted use, you will need to obtain permission directly from the copyright holder. To view a copy of this license, visit http://creativecommons. org/licenses/by-nc-sa/4.0/.

\section{References}

1. Calbet A. Mesozooplankton grazing effect on primary production: a global comparative analysis in marine ecosystems. Limnol Oceanogr. 2001;46:1824-30.

2. Calbet A, Landry MR. Phytoplankton growth, microzooplankton grazing, and carbon cycling in marine systems. Limnol Oceanogr. 2004:49:51-57.

3. Long JD, Smalley GW, Barsby T, Anderson JT, Hay ME. Chemical cues induce consumer-specific defenses in a bloom-forming 
marine phytoplankton. Proc Natl Acad Sci USA. 2007;104:10512-7.

4. Selander E, Jakobsen HH, Lombard F, Kiørboe T. Grazer cues induce stealth behavior in marine dinoflagellates. Proc Natl Acad Sci USA. 2011;108:4030-4.

5. Selander E, Thor P, Toth G, Pavia H. Copepods induce paralytic shellfish toxin production in marine dinoflagellates. Proc R Soc B Biol Sci. 2006;273:1673-80.

6. Tammilehto A, Nielsen TG, Krock B, Møller EF, Lundholm N. Induction of domoic acid production in the toxic diatom Pseudonitzschia seriata by calanoid copepods. Aquat Toxicol (Amsterdam, Netherlands). 2015;159:52-61.

7. Bergkvist J, Thor P, Jakobsen HH, Wängberg S-Å, Selander E. Grazer-induced chain length plasticity reduces grazing risk in a marine diatom. Limnol Oceanogr. 2012;57:318-24.

8. Bjaerke O, Jonsson PR, Alam A, Selander E. Is chain length in phytoplankton regulated to evade predation? J Plankton Res. 2015;37:1110-9.

9. Suraci JP, Clinchy M, Dill LM, Roberts D, Zanette LY. Fear of large carnivores causes a trophic cascade. Nat Commun. 2016;7:10698.

10. Kats LB, Dill LM. The scent of death: chemosensory assessment of predation risk by prey animals. Écoscience. 1998;5:361-94.

11. Werner EE, Peacor SD. A review of trait-mediated indirect interactions in ecological communities. Ecology. 2003;84:1083-1100.

12. Stibor H, Vadstein O, Diehl S, Gelzleichter A, Hansen $T$, Hantzsche F, et al. Copepods act as a switch between alternative trophic cascades in marine pelagic food webs. Ecol Lett. 2004;7:321-8.

13. Yang I, Selander E, Pavia H, John U. Grazer-induced toxin formation in dinoflagellates: a transcriptomic model study. Eur J Phycol. 2011;46:66-73.

14. Wohlrab S, Tillmann U, Cembella A, John U. Trait changes induced by species interactions in two phenotypically distinct strains of a marine dinoflagellate. ISME J. 2016;10:2658-68.

15. Ianora A, Miralto A. Toxigenic effects of diatoms on grazers, phytoplankton and other microbes: a review. Ecotoxicol Lond Engl. 2010;19:493-511.

16. Saravanan V, Godhe A. Genetic heterogeneity and physiological variation among seasonally separated clones of Skeletonema marinoi (Bacillariophyceae) in the Gullmar Fjord, Sweden. Eur J Phycol. 2010;45:177-90.

17. Gerecht A, Romano G, Ianora A, d'Ippolito G, Cutignano A, Fontana A. Plasticity of oxylipin metabolism among clones of the marine diatom Skeletonema marinoi (bacillariophyceae). J Phycol. 2011;47:1050-6.

18. Di Dato V, Orefice I, Amato A, Fontanarosa C, Amoresano A, Cutignano A, et al. Animal-like prostaglandins in marine microalgae. ISME J. 2017;11:1722-6.

19. Ribalet F, Berges JA, Ianora A, Casotti R. Growth inhibition of cultured marine phytoplankton by toxic algal-derived polyunsaturated aldehydes. Aquat Toxicol (Amsterdam, Netherlands). 2007;85:219-27.

20. Vardi A, Bidle KD, Kwityn C, Hirsh DJ, Thompson SM, Callow $\mathrm{JA}$, et al. A diatom gene regulating nitric-oxide signaling and susceptibility to diatom-derived aldehydes. Curr Biol. 2008;18:895-9.

21. Bockaert J, Pin JP. Molecular tinkering of G protein-coupled receptors: an evolutionary success. EMBO J. 1999;18:1723-9.

22. Guillard RRL. Culture of phytoplankton for feeding marine invertebrates. In: Smith WL, Chanley MH, editors. Culture of marine invertebrate animals. New York, NY, US: Springer; 1975. pp. 29-60.

23. Basu S, Patil S, Mapleson D, Russo MT, Vitale L, Fevola C, et al. Finding a partner in the ocean: molecular and evolutionary bases of the response to sexual cues in a planktonic diatom. New Phytol. 2017;215:140-56.

24. Bolger AM, Lohse M, Usadel B. Trimmomatic: a flexible trimmer for Illumina sequence data. Bioinforma Oxf Engl. 2014;30:2114-20.

25. Haas BJ, Papanicolaou A, Yassour M, Grabherr M, Blood PD, Bowden J, et al. De novo transcript sequence reconstruction from RNA-seq using the Trinity platform for reference generation and analysis. Nat Protoc. 2013;8:1494-512.

26. Musacchia F, Basu S, Petrosino G, Salvemini M, Sanges R. Annocript: a flexible pipeline for the annotation of transcriptomes able to identify putative long noncoding RNAs. Bioinforma Oxf Engl. 2015;31:2199-201.

27. Langmead B, Trapnell C, Pop M, Salzberg SL. Ultrafast and memory-efficient alignment of short DNA sequences to the human genome. Genome Biol. 2009;10:R25.

28. Tamura K, Stecher G, Peterson D, Filipski A, Kumar S. MEGA6: molecular evolutionary genetics analysis version 6.0. Mol Biol Evol. 2013;30:2725-9.

29. Posada D, Crandall KA. Selecting the best-fit model of nucleotide substitution. Syst Biol. 2001;50:580-601.

30. Bylesjö M, Rantalainen M, Cloarec O, Nicholson JK, Holmes E, Trygg J. OPLS discriminant analysis: combining the strengths of PLS-DA and SIMCA classification. J Chemom. 2006;20:341-51.

31. Trygg J, Wold S. Orthogonal projections to latent structures (OPLS). J Chemom. 2002;16:119-28.

32. Haug K, Salek RM, Conesa P, Hastings J, de Matos P, Rijnbeek $\mathrm{M}$, et al. MetaboLights--an open-access general-purpose repository for metabolomics studies and associated meta-data. Nucleic Acids Res. 2013;41:D781-786.

33. Port JA, Parker MS, Kodner RB, Wallace JC, Armbrust EV, Faustman EM. Identification of $G$ protein-coupled receptor signaling pathway proteins in marine diatoms using comparative genomics. BMC Genom. 2013;14:503.

34. Di Dato V, Musacchia F, Petrosino G, Patil S, Montresor M, Sanges R, et al. Transcriptome sequencing of three Pseudonitzschia species reveals comparable gene sets and the presence of nitric oxide synthase genes in diatoms. Sci Rep. 2015;5:12329.

35. Gruber A, Rocap G, Kroth PG, Armbrust EV, Mock T. Plastid proteome prediction for diatoms and other algae with secondary plastids of the red lineage. Plant J. 2015;81:519-28.

36. Rogato A, Amato A, Iudicone D, Chiurazzi M, Ferrante MI, d'Alcalà MR. The diatom molecular toolkit to handle nitrogen uptake. Mar Genom. 2015;24:95-108. Pt 1

37. Corner EDS, Newell BS. On the nutrition and metabolism of zooplankton IV. The forms of nitrogen excreted by Calanus. J Mar Biol Assoc U K. 1967;47:113-20.

38. Huysman MJJ, Vyverman W, De Veylder L. Molecular regulation of the diatom cell cycle. J Exp Bot. 2014;65:2573-84.

39. Huysman MJ, Martens C, Vandepoele K, Gillard J, Rayko E, Heijde M, et al. Genome-wide analysis of the diatom cell cycle unveils a novel type of cyclins involved in environmental signaling. Genome Biol. 2010;11:R17.

40. Amin SA, Hmelo LR, van Tol HM, Durham BP, Carlson LT, Heal KR, et al. Interaction and signalling between a cosmopolitan phytoplankton and associated bacteria. Nature. 2015;522:98-101.

41. Bergkvist J, Selander E, Pavia H. Induction of toxin production in dinoflagellates: the grazer makes a difference. Oecologia. 2008;156:147-54.

42. Barofsky A, Simonelli P, Vidoudez C, Troedsson C, Nejstgaard JC, Jakobsen HH, et al. Growth phase of the diatom Skeletonema marinoi influences the metabolic profile of the cells and the selective feeding of the copepod Calanus spp. J Plankton Res. 2010;32:263-72. 
43. Vidoudez C, Pohnert G. Comparative metabolomics of the diatom Skeletonema marinoi in different growth phases. Metabolomics. 2012;8:654-69.

44. Ashworth J, Coesel S, Lee A, Armbrust EV, Orellana MV, Baliga NS. Genome-wide diel growth state transitions in the diatom Thalassiosira pseudonana. Proc Natl Acad Sci USA. 2013;110:7518-23.

45. Bjarnadóttir TK, Gloriam DE, Hellstrand SH, Kristiansson H, Fredriksson R, Schiöth HB. Comprehensive repertoire and phylogenetic analysis of the $\mathrm{G}$ protein-coupled receptors in human and mouse. Genomics. 2006;88:263-73.

46. Kobilka BK. G protein coupled receptor structure and activation. Biochim Biophys Acta BBA - Biomembr. 2007;1768:794-807.

47. Mukhopadhyay S, Jackson PK. The tubby family proteins. Genome Biol. 2011;12:225.

48. Zurzolo C, Bowler C. Exploring bioinorganic pattern formation in diatoms. A story of polarized trafficking. Plant Physiol. 2001;127:1339-45.

49. Maumus F, Allen AE, Mhiri $\mathrm{C}, \mathrm{Hu} \mathrm{H}$, Jabbari $\mathrm{K}$, Vardi A, et al. Potential impact of stress activated retrotransposons on genome evolution in a marine diatom. BMC Genom. 2009;10:624.

50. Vardi A, Formiggini F, Casotti R, De Martino A, Ribalet F, Miralto A, et al. A stress surveillance system based on calcium and nitric oxide in marine diatoms. PLoS Biol. 2006;4:e60.

51. Pohnert G. Wound-activated chemical defense in unicellular planktonic algae. Angew Chem Int Ed. 2000;39:4352-4.

52. Tanaka T, Maeda Y, Veluchamy A, Tanaka M, Abida H, Maréchal E, et al. Oil accumulation by the oleaginous diatom Fistulifera solaris as revealed by the genome and transcriptome. Plant Cell. 2015;27:162-76.

53. Jouhet J, Lupette J, Clerc O, Magneschi L, Bedhomme M, Collin $\mathrm{S}$, et al. LC-MS/MS versus TLC plus GC methods: consistency of glycerolipid and fatty acid profiles in microalgae and higher plant cells and effect of a nitrogen starvation. PLoS ONE. 2017;12: e0182423.

54. Abida H, Dolch L-J, Meï C, Villanova V, Conte M, Block MA, et al. Membrane glycerolipid remodeling triggered by nitrogen and phosphorus starvation in Phaeodactylum tricornutum. Plant Physiol. 2015;167:118-36.

55. Kim E-S, Choi E, Kim Y, Cho K, Lee A, Shim J, et al. Dual positional specificity and expression of non-traditional lipoxygenase induced by wounding and methyl jasmonate in maize seedlings. Plant Mol Biol. 2003;52:1203-13.

56. Mock T, Samanta MP, Iverson V, Berthiaume C, Robison M, Holtermann $\mathrm{K}$, et al. Whole-genome expression profiling of the marine diatom Thalassiosira pseudonana identifies genes involved in silicon bioprocesses. Proc Natl Acad Sci USA. 2008; 105:1579-84.

57. Tesson B, Masse S, Laurent G, Maquet J, Livage J, MartinJézéquel V, et al. Contribution of multi-nuclear solid state NMR to the characterization of the Thalassiosira pseudonana diatom cell wall. Anal Bioanal Chem. 2008;390:1889-98.

58. Sarno D, Kooistra WHCF, Medlin LK, Percopo I, Zingone A. Diversity in the genus Skeletonema (bacillariophyceae). II. An assessment of the taxonomy of $S$. Costatum-like species with the description of four new species. J Phycol. 2005;41: $151-76$.

59. Round, FE, Crawford, RM, Mann, DG. Diatoms biology and morphology of the genera. Cambridge: Cambridge University Press; 2007.

60. Ehrlich H, Simon P, Carrillo-Cabrera W, Bazhenov VV, Botting JP, Ilan M, et al. Insights into chemistry of biological materials: newly discovered silica-aragonite-chitin biocomposites in demosponges. Chem Mater. 2010;22:1462-71.

61. Ogasawara W, Shenton W, Davis SA, Mann S. Template mineralization of ordered macroporous chitin-silica composites using a cuttlebone-derived organic matrix. Chem Mater. 2000;12:2835-7.

62. Trussell GR, Schmitz OJ. Species functional traits, trophic control and the ecosystem consequences of adaptive foraging in the middle of food chains. In: Ohgushi T, Schmitz OJ, Holt RD, editors. Trait-mediated indirect interactions: ecological and evolutionary perspectives. Cambridge: Cambridge University Press; 2012, pp. 324-38. 